Reprod. Nutr. Dévelop., 1987, 27 (1 B), 289-290.

\title{
Mise au point d'une fistule iléale chez le lapin adulte. Caractérisation des digesta prélevés
}

T. GIDENNE, T. BOUYSSOU $\left({ }^{*}\right)$

Laboratoire de Recherches sur l'Elevage du Lapin, I.N.R.A., B.P. 27, 31326 Castanet Tolosan Cedex.

(*) Laboratoire de Physiologie, Ecole Nationale Vétérinaire Chemin des Capelles, 31026 Toulouse Cedex.

Summary. The terminal ileum in five adult rabbits was cannulated; four animals still have a functional cannula 6 months after the operation. This method can provide a convenient model for digestibility studies.

Chez le lapin, la ration est d'abord dégradée par les sucs digestifs dans l'intestin grêle ; elle est ensuite fermentée par la flore caecocolique. Une mesure de digestibilité à la fin de l'iléon permettrait d'évaluer l'importance de ces deux étapes de la digestion. Dans ce but, nous avons établi une fistule iléale permanente chez le lapin et nous avons cherché à connaître les limites de cette technique : tolérance de la canule par l'animal et validité des prélèvements au plan quantitatif et qualitatif.

Matériel et méthodes. Trois femelles et deux mâles adultes reçoivent ad libitum un aliment commercial ( $14 \%$ de protéines et $16 \%$ de cellulose brute). Après un jeûne de $24 \mathrm{~h}$, une laparotomie est effectuée sous anesthésie générale (pentobarbital $20 \mathrm{mg} / \mathrm{kg}$ ). Une canule de Pyrex en $T$ (diamètre interne : $4 \mathrm{~mm}$ et interne : $7 \mathrm{~mm}$ ) est placée à $5 \mathrm{~cm}$ de la valvule iléocæcale, en position abdominale. Les prélèvements débutent deux semaines après l'opération. Ils sont repartis en 8 récoltes sur l'ensemble du nycthémère (tabl. 1); ce schéma est répété lors d'une seconde série de prélèvements. Chaque lapin fait l'objet de deux à trois récoltes hebdomadaires d'une heure chacune. Pour cela, l'animal est mis en contention dans un " hamac » afin de récolter le contenu iléal par gravité. Les quantités de digesta prélevées permettent les mesures suivantes : matière sèche (MS), matières azotées (MAT), $\mathrm{pH}$, acides gras volatils totaux (AGV) (série 1), ammoniaque $\left(\mathrm{NH}_{3}\right)$.

Résultats et discussion. La durée de cet essai a été de 10 semaines. Seul un animal sur 7 opérés est mort par occlusion intestinale à la suite de l'opération. Quatre animaux portent encore une canule fonctionnelle 6 mois plus tard. Deux semaines après l'opération, les animaux retrouvent leur poids et leur consommation initiale. Seules les teneurs en MAT des prélèvements diffèrent entre les deux séries de mesures. Les valeurs moyennes des poids frais récoltés diffèrent $(P<0,01)$ en fonction de l'heure de prélèvement. Ce phénomène peut s'expliquer par les variations nycthémérales de l'ingestion, ainsi que par des variations de rétention gastrique ou intestinale. En accord avec les données bibliographiques (Leng et Hornicke, 1975 ; Gidenne et Poncet, 1985), les teneurs moyennes en MAT $\left(\mathrm{NH}_{3}\right)$ ou AGV ne varient pas significativement en fonction de l'heure (tabl. 1). Dans le cas du pH ou des teneurs en MS, la présence d'interactions entre les effets individus et heure ne permet pas d'analyser les écarts enregistrés. 
Une maîtrise encore imparfaite de la technique de récolte peut être une cause des variations intra-individus parfois élevées (tabl. 1). D'autres sources de variations peuvent masquer un effet éventuel de l'heure de prélèvement sur la composition des contenus iléaux : par exemple, le non synchronisme des cycles digestifs individuels (Jilge, 1974), le poids $(2,4$ à $4 \mathrm{~kg})$ ou le sexe des animaux.

TABL. 1. - Caractéristiques movennes $(n=10)$ des contenus iléaux. Evolution nycthémérale.

\begin{tabular}{|c|c|c|c|c|c|c|c|c|c|c|c|}
\hline Heure & 9 & 12 & 15 & 18 & 21 & 24 & 3 & 6 & $\bar{x}$ & $\begin{array}{c}F \\
\text { heure }\end{array}$ & $\begin{array}{l}\text { CV \% } \\
\text { intra } \\
\text { individu }\end{array}$ \\
\hline $\begin{array}{l}\text { Poids frais }(\mathrm{g}) \\
\mathrm{pH} \\
\text { MS (\%) } \\
\text { MAT (\% MS) } \\
\text { AGV (mM/l) } \\
\mathrm{NH}_{3}(\mathrm{mM} / \mathrm{l})\end{array}$ & $\begin{array}{c}24,6 \\
7,94 \\
10,5 \\
17,2 \\
7,7 \\
22,7\end{array}$ & $\begin{array}{c}20,1 \\
7,93 \\
11,0 \\
17,8 \\
8,4 \\
18,0\end{array}$ & $\begin{array}{c}14,4 \\
8,16 \\
10,2 \\
17,1 \\
14,1 \\
25,4\end{array}$ & $\begin{array}{c}18,4 \\
8,08 \\
11,0 \\
17,5 \\
10,4 \\
37,8\end{array}$ & $\begin{array}{c}22,6 \\
8,03 \\
9,9 \\
17,7 \\
8,7 \\
22,8\end{array}$ & $\begin{array}{c}14,7 \\
7,97 \\
10,0 \\
16,9 \\
10,5 \\
18,3\end{array}$ & $\begin{array}{c}21,2 \\
7,87 \\
9,8 \\
16,2 \\
6,8 \\
18,3\end{array}$ & $\begin{array}{c}18,4 \\
7,96 \\
9,9 \\
14,9 \\
7,1 \\
22,6\end{array}$ & $\begin{array}{c}19,4 \\
7,99 \\
10,3 \\
16,7 \\
9,0 \\
23,2\end{array}$ & $\begin{array}{r}* * \\
* \\
* \\
N S \\
N S \\
N S\end{array}$ & $\begin{array}{r}30,7 \\
2,1 \\
9,1 \\
21,9 \\
41,9 \\
63,6\end{array}$ \\
\hline
\end{tabular}

En conclusion, cette expérience montre qu'il est possible d'établir une fistule iléale chez le lapin capable de fournir un modèle d'étude de digestibilité convenable. Les variations mesurées lors de ces premiers essais incitent à standardiser la technique de récolte, à éviter un mélange des prélèvements entre individus, et à maintenir une répartition des prélèvements sur l'ensemble du nycthémère.

Gidenne T., Poncet C., 1985. Ann. Zootech., 34, 429-445.

Jilge B., 1974, Labor. Anim., 8, 337-346.

Leng E., Hornicke H., 1975. 'Z. Versuchstierkd., 17, 285-299. 